\title{
Multiple Scale Analysis of the Forced Response of a Mistuned Bladed Disk With Nonlinear Friction Forces
}

\author{
Juan A. Martin* and Carlos Martel ${ }^{\dagger}$ \\ Universidad Politécnica de Madrid. Pza. Cardenal Cisneros 3, 28040. Madrid. Spain
}

\begin{abstract}
The unavoidable small blade-to-blade variations in a turbomachinery bladed disk are know as "mistuning". When compared with the ideal, "tuned", bladed-disk, the mistuning can produce a considerable increase of the vibratory forced response level of the blades. This situation can lead to high cycle fatigue failure, and, therefore, the correct estimation of the vibratory response is of crucial importance for the prediction of the operative life span of the bladed disk. The computation of the final amplitude of the limit cycle oscillation requires to solve a quite complicated problem; the mistuned bladed disk is not cyclic symmetric, and, consequently, the complete bladed disk has to be considered, increasing dramatically the computational cost of the numerical simulations. For this reason we discuss the possibility of applying an asymptotic multiple scale analysis to derive a simplified model that can be used to analyze the characteristics of the final vibration states at a much lower computational cost. The key idea for the simplification is the fact that all significant effects present (forcing, nonlinear friction damping, and mistuning) are, in most practical situations, small effects, that develop in a time scale that is much longer than that associated with the natural elastic vibration frequency of the tuned systen. In this paper the bladed disk is described using a mass-spring system with nonlinear friction, and we consider the frequent case of forcing a blade dominated modal family, where all modes have very similar vibration frequencies. The derivation of the asymptotically simplified model is explained in detail, and the validity of its results is verified against the results from the original mass-spring model, for both tuned and mistuned configurations.
\end{abstract}

\section{Introduction}

$\mathrm{T}$ HE ever incresing demand for higher efficiency in modern turbomachinery moves the designs towards higher loadings and thinner blades. This makes the bladed disks more susceptible to experience aeromechanic vibrations. The sources of blade vibration energy (flutter or forced response) must be counterbalanced by the dissipation of nonlinear friction forces at the contact interfaces between blade and disk, and this balance determines the final vibration amplitude of the blade. The amplitude of the blade vibration is of extreme practical importance in order to estimate the blade operative life span, as it constitutes a key information for the prediction of the level of high cycle fatigue (HCF).

In a turbomachine, the upstream stator generates a pulsating aerodynamic force over the rotor, that can be considered to be purely harmonic in time. The vibration of the blade and the disk can be accurately modelled using linear elasticity, but the friction forces at the contact interfaces follow a nonlinear model $[1,2]$. The vibration response of the blades is no longer composed of a single harmonic because the nonlinearity generates multiple harmonics that necessarily have to be taken into account.

The harmonic balance method (HBM) is normally used for the calculation of the blade vibration in the frequency plane (see, f.i., [3-5]). The HBM requieres to solve a large system of nonlinear algebraic equations. The number of equations grows proportionally to the number of harmonics that are included in the description, and, in order to properly represent the highly nonlinear behaviour of the friction forces, a considerable number of harmonics has to be kept: despite of the fact that the nonlinearities are present only in a small fraction of the total number degrees of freedom.

In the case of a tuned rotor, the HBM calculation can be reduced to a single sector with appropriate phase lag boundary conditions, but, if mistuning is taken into account, the cyclic symmetry of the problem is lost, and consequently the complete bladed disk has to be considered. The computational cost of the mistuned cased is thus dramatically

\footnotetext{
*Assistant Lecturer, Department of Applied Mathematics to Aerospace Engineering, Universidad Politécnica de Madrid, juanangel.nartin@ upm.es ORCID iD: https://orcid org/0000-0003-0148-1398

${ }^{\dagger}$ Full Professor. Department of Applied Mathematics to Aerospace Engineering, Universidad Politécnica de Madrid. cartos.martel@upm.es
} 
increased, making it imperative to look for methods that allow for the reduction of the problem complexity keeping a satisfactory level of accuracy.

In previous works the multiple scale method has been successfully applied to the study of the flutter vibration amplitude saturation by nonlinear friction forces [6], and to the calculation of the friction effects on the forced response of a mistuned bladed disk for the case of exciting an isolated mode [7], where only two travelling waves (TW) are present in the response of the system.

In this paper, the multiple scale method is applied to derive a simplified model for a mistuned bladed disk in the more complex situation of forcing a mode that belongs to a blade dominated modal family. All modes in the family have very similar frequencies and, therefore, the mistuning coupling effect gives rise to a vibration response that can contain all TW. A mass-spring model with 2 degrees of freedom (DOF) per sector (blade and friction displacement) is used to represent the mistuned bladed disk. A very reduced asymptotic model is derived using multiple scale techniques $[8,9]$ for both the tuned and mistuned cases, and the results from the simplified model are compared with those from the original mass-spring model.

\section{Mass-spring model}

The mass-spring model (sketched in Fig. 1) describes the forcing of a nearly flat modal family of a bladed disk with mistuning and nonlinear friction effects. It contains a total of $N$ sectors, with 2 DOF per sector: the displacement of the blade, $x_{j}$, and the microslip friction displacement at the fir-tree, $y_{j}$.

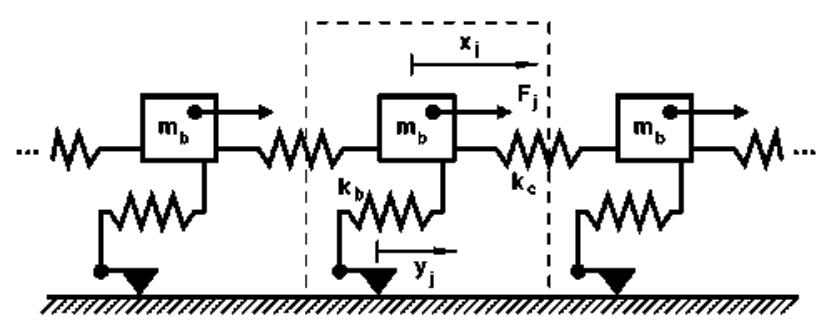

Fig. 1 Simplified mass-spring model of a bladed disk with friction elements.

The corresponding equations of motion for the tuned case can be written as:

$$
\begin{gathered}
m_{b} \frac{d^{2} x_{j}}{d t^{2}}+k_{b}\left(x_{j}-y_{j}\right)+k_{c}\left(2 x_{j}-x_{j+1}-x_{j-1}\right)+c \frac{d x_{j}}{d t}=F \mathrm{e}^{\mathrm{i} \omega t+\mathrm{i} E O \frac{2 \pi}{N} j}+\mathrm{c.c} . \\
m_{f} \frac{d^{2} y_{j}}{d t^{2}}+k_{b}\left(y_{j}-x_{j}\right)+F_{f}\left(y_{j}\right)=0 \text { for } j=1, \ldots, N
\end{gathered}
$$

where $m_{b}$ is the blade mass, $k_{b}$ is the blade stiffness, $c$ is a small material damping, and $k_{c}$ represents the stiffness of the coupling with the adjacent sectors (i.e., the effect of the coupling of the blades through the disk). The forcing takes the form of a traveling wave (TW) with amplitude $F$, engine order $E O$, and frequency $\omega$ (c.c. stands for the complex conjugate so the real expression for the forcing is $2 F \cos \left(\omega t+E O \frac{2 \pi}{N} j\right)$ ). The mass of the friction degree of freedom is $m_{f}$, and $F_{f}\left(y_{j}\right)$ is the friction force that depends only on the microslip friction displacement at the fir-tree $y_{j}$.

For the friction force $F_{f}$, we make use of the Olofsson microslip model [10]. The magnitude of the friction force for a loading-unloading cycle can be expressed as:

$$
F_{f}(y)=F^{*} \pm F(y)
$$

with $F^{*}$ taken from the preceding loading-unloading switching point, the + sign corresponds to the loading phase, and to the unloading; as represented in Fig. 2. The increment of the friction force $F(y)$ is given by:

$$
F(y)=2 F_{c}\left(1-\left(1-\frac{\left|y-y^{*}\right|}{2 y_{c}}\right)^{\frac{5}{2}}\right)
$$

where $y^{*}$ denotes the displacement at the previous turning point. The microslip regime is delimited by the microslip characteristic force $F_{c}$ and displacement $y_{c}$. There is, therefore, a maximum increment of friction force and displacement of $2 F_{c}$ and $2 y_{c}$ inside the microslip regime. 


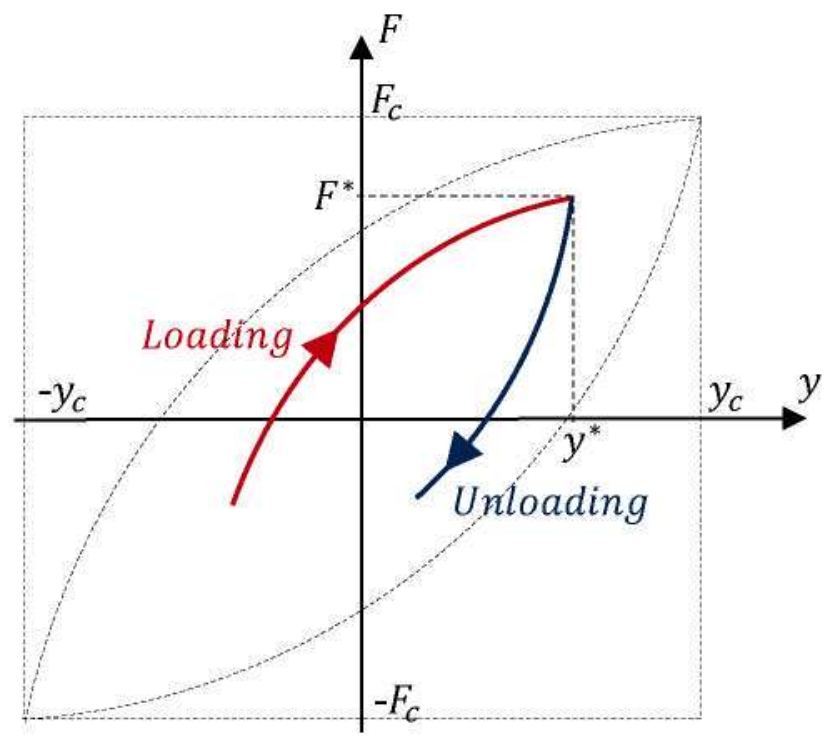

Fig. 2 Olofsson model: friction force for a loading-unloading cycle, with the limit of the microslip regime marked with dotted lines.

The Olofsson nonlinear friction force model involves two effects: the first one is the characteristic stiffness measured as $F_{c} / y_{c}$, i.e., the slope of the straight line connecting the origin with $\left(y_{c}, F_{c}\right)$ (with the friction forces acting as a linear spring); and the second one, the hysteresis effect resulting in a damping action as a consequence of the difference between the loading and unloading curves.

The system (1) is made nondimensional in order to easily compare the relative importance of the different existent effects. Time is made nondimensional using the blade alone frequency:

$$
T=t \omega_{b}=t \sqrt{\frac{k_{b}}{m_{b}}}
$$

the microslip displacement and force with their characteristic values

$$
\tilde{y}=y / y_{c}, \quad \tilde{F}_{f}(\tilde{y}) / F_{c},
$$

the blade displacement with its characteristic value, $x_{c}$, coming from the balance of the friction force with the blade motion induced force at the microslip element $\left(F_{c}=k_{b} x_{c}\right)$

$$
\tilde{x}=x / x_{c}=x /\left(F_{c} / k_{b}\right),
$$

the forcing magnitude with the forces produced by the microslip displacement on the blade

$$
f=F /\left(k_{b} y_{c}\right),
$$

and, finally, the forcing frequency with the blade alone frequency

$$
\tilde{\omega}=\omega / \omega_{b},
$$

The resulting nondimensional system of equations takes the form

$$
\begin{gathered}
\frac{d^{2} \tilde{x}_{j}}{d T^{2}}+\left(\tilde{x}_{j}-\theta \tilde{y}_{j}\right)+\theta k\left(2 \tilde{x}_{j}-\tilde{x}_{j+1}-\tilde{x}_{j-1}\right)+\theta c \frac{d \tilde{x}_{j}}{d T}=\theta f \mathrm{e}^{\mathrm{i} \hat{\omega} T+\mathrm{i} E O \frac{2 \pi}{N} j}+\text { c.c. } \\
\theta \gamma \frac{d^{2} \tilde{y}_{j}}{d T^{2}}+\left(\theta \tilde{y}_{j}-\tilde{x}_{j}\right)+\tilde{F}_{f}\left(\tilde{y}_{j}\right)=0 \quad \text { for } j=1, \ldots, N
\end{gathered}
$$


where the nondimensional parameter

$$
\gamma=\frac{m_{f}}{m_{b}}
$$

is the ratio between the microslip DOF and blade DOF masses, and $\theta$ is the ratio between microslip and blade displacements

$$
\theta=\frac{y_{c}}{x_{c}}=\frac{k_{b}}{\left(F_{c} / y_{c}\right)}
$$

which is typically very small, $\theta \ll 1$. For the case analyzed in this paper, where a complete blade dominated modal family is considered, the ratio between blade and coupling stiffness is also taken to be very small $\left(\frac{k_{c}}{k_{b}}=\theta k \ll 1\right)$, in order to represent a small blade coupling effect induced by the disk.

The limit $\theta \ll 1$ indicates that the effect of friction is very small compared to the elastic forces. This means that the friction forces have to balance with the external forcing to saturate the growth of the vibration amplitude. The remaining effects in the first equation in system (4), corresponding to the blade motion equations, are all conservative, and cannot dissipate the energy injected into the system by the forcing.

In the next sections, an asymptotic perturbative procedure based on the small parameter $\theta$ will be developed using a multiple scale technique (see $[8,9]$ for a more complete description this methodology). It will provide a very simplified model for the dynamics of the mass-spring system, Eq. (4), valid for both tuned and mistuned cases.

\section{Tuned Response}

The common case where the friction effects are much smaller that the elastic forces is represented as the asymptotic limit of very small $\theta$. The forced response of the mass spring system, modeled with the set of equations Eq. (4),) corresponds, in first approximation, to the elastic blade vibration mode without friction and forcing. The friction effect is then connected to the small displacement at the contact interfaces, generating an adjustment of the purely elastic vibration characteristics. The final value of the amplitude of the blade vibration amplitude is obtained after the system completes a large number of elastic oscillation periods, which are required to see the action of the small effects associated with the forcing and the friction.

The multiple scales method (see [8,9]) is employed to approximate the dynamics of the system (4), which, due to the existence of a small parameter, exhibits two very disparate time scales: fast (elastic) and slow (disk coupling, forcing and friction). Two time scale variables are introduced and handled as if they were independent, then the solution is expanded in powers of the small parameter, and finally the slow time evolution is obtained by making use of the so-called "solvability conditions" to eliminate the possibility of unbounded in time components in the final solution.

The solution of system (4) is expanded as

$$
\begin{aligned}
& \tilde{x}_{j}=\tilde{x}_{j}^{0}(T, \tau)+\theta \tilde{x}_{j}^{1}(T, \tau)+\ldots \\
& \tilde{y}_{j}=\ddot{y}_{j}^{0}(T, \tau)+\theta \tilde{y}_{j}^{1}(T, \tau)+\ldots
\end{aligned}
$$

where $T \sim 1$ is the fast elastic oscillation time, and $\tau=T \theta$ is the slow time connected to the small coupling, friction and forcing effects.

The expansions (5) are introduced into the blade motion system (4), and the following expression is obtained for the first order

$$
\begin{aligned}
\tilde{x}_{j T T}^{0}+\tilde{x}_{j}^{0} & =0 \\
\tilde{x}_{j}^{0} & =F_{f}\left(\tilde{y}_{j}^{0}\right) / F_{c} .
\end{aligned}
$$

Equation (6) can be easily solved to give

$$
\tilde{x}_{j}^{0}=X_{j}(\tau) \mathrm{e}^{i T}+c . c .,
$$

meaning that, in the fast time scale $T$, all airfoils vibrate with the elastic frequency, while the amplitude of those oscillations, $X_{j}$, is modulated in the slow time scale $\tau$. Therefore, at first order, the motion of the blades corresponds to the natural oscillation with the blade alone frequency $(\omega=1)$ and with the microsplip DOF stuck.

If the disk coupling is retained, the natural frequencies of the TW modes are given by the expression 


$$
\omega_{m}=\sqrt{1+2 \frac{k_{c}}{k_{b}}\left(1-\cos \left(m \frac{2 \pi}{N}\right)\right)}
$$

where the TW index $m$ goes from 0 to $N-1$, and the frequency of the TW modes, $\omega_{m}$, grows from 1 to $\sqrt{1+4 \frac{k_{c}}{k_{b}}}$. In the case of small disk coupling, the ratio between blade and coupling stiffness $\left(\frac{k_{c}}{k_{b}}\right)=\theta k$ is very small, and then both limits are almost the same, and all mode frequencies are very similar and equal to 1 (blade alone frequency). Figure 3 shows the tuned natural vibration frequencies versus the TW index $m$ for a small value of $\theta=0.001$. The plot shows the typical family of blade-dominated modes, evidenced by the almost horizontal line.

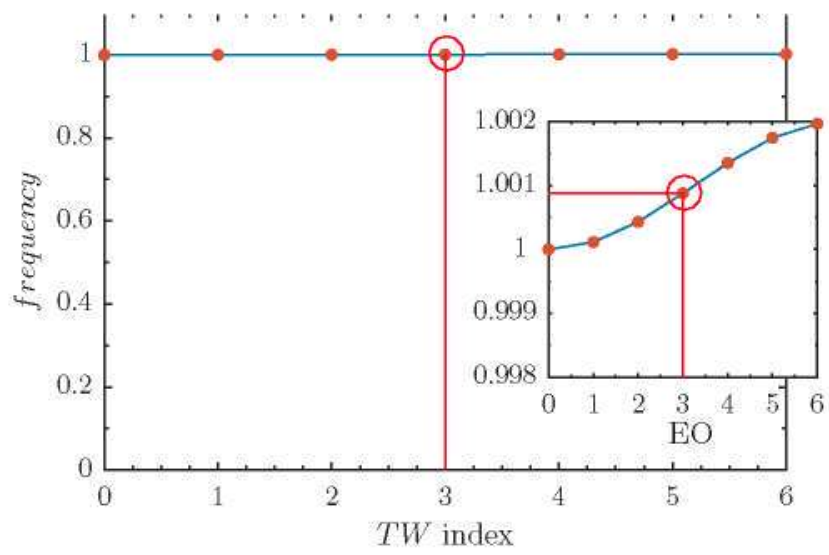

Fig. 3 Tuned natural vibration frequencies of a nearly flat modal family (engine order $E O$ forcing indicated).

The left hand side of the second equation of the system (6), $\tilde{x}_{j}^{0}$ is now determined, and can be employed to compute the first order of the microslip DOF displacement $\tilde{y}_{j}^{0}$. A real periodic function in $T$ is obtained, which can be expressed in Fourier series as

$$
\tilde{y}_{j}^{0}=P\left(T, X_{j}\right)=\sum_{k=1}^{\infty} P_{k}\left(X_{j}\right) \mathrm{e}^{i k T}+\text { c.c. }
$$

It is a periodic function with the same $2 \pi$ period as the blades (7) but with all harmonics because of the nonlinear character of the friction force.

The next order approximation, corresponding to the slow time scale for the airfoil displacements, is achieved after taking the expressions (5) into Eq. (4) and retaining the next order terms

$$
\frac{\partial^{2} \tilde{x}_{j}^{1}}{\partial T^{2}}+2 \frac{\partial^{2} \tilde{x}_{j}^{0}}{\partial T \partial \tau}+\tilde{x}_{j}^{1}-\tilde{y}_{j}^{0}+k\left(2 \tilde{x}_{j}^{0}-\tilde{x}_{j+1}^{0}-\tilde{x}_{j-1}^{0}\right)+c \frac{\partial \tilde{x}_{j}^{0}}{\partial T}=f \mathrm{e}^{\mathrm{i} \tilde{\omega} T+\mathrm{i} E O \frac{2 \pi}{N} j}+\mathrm{c} . \mathrm{c} .
$$

Now, making use of the expressions for $\tilde{x}_{j}^{0}(6)$ and $\tilde{y}_{j}^{0}(8)$, the preceding Eq. (9) can be re-formulated as

$$
\frac{\partial^{2} \tilde{x}_{j}^{1}}{\partial T^{2}}+\tilde{x}_{j}^{1}=-2 \mathrm{i} \frac{\partial X_{j}}{\partial \tau} \mathrm{e}^{\mathrm{i} T}+\sum_{k=1}^{\infty} P_{k}\left(X_{j}\right) \mathrm{e}^{\mathrm{i} k T}-\left(k\left(2 X_{j}-X_{j+1}-X_{j-1}\right)+\mathrm{i} c X_{j}\right) \mathrm{e}^{\mathrm{i} T}+f \mathrm{e}^{\mathrm{i} T} \mathrm{e}^{\mathrm{i} \Delta \omega \theta T+\mathrm{i} E O \frac{2 \pi}{N} j}+c . c .
$$

where the forcing frequency has also been expanded as $\tilde{\omega}=1+\theta \Delta \omega$, to describe a forcing near the resonant frequency $\omega_{0}=1$.

A bounded evolution for $\tilde{x}_{j}^{1}(T, \tau)$ is only possible if no terms proportional to $\mathrm{e}^{\mathrm{i} T}$ appear in the right hand side of the previous expression, see $[8,9]$. This constraint provides the evolution equation for the blade amplitudes $X_{j}$ in the slow time scale $\tau$

$$
2 \mathrm{i} \frac{\partial X_{j}}{\partial \tau}=P_{1}\left(X_{j}\right)-k\left(2 X_{j}-X_{j+1}-X_{j-1}\right)-\mathrm{i} c X_{j}+f \mathrm{e}^{\mathrm{i} \Delta \omega \tau+\mathrm{i} E O \frac{2 \pi}{N} j}+\text { c.c. }
$$


The first Fourier coefficient $P_{1}$ of $\tilde{y}_{j}^{0}$ can be expressed in terms of the complex friction function $Q$

$$
P_{1}\left(X_{j}\right)=Q\left(\left|X_{j}\right|\right) X_{j},
$$

which comprises all the effect from the nonlinear friction forces: the real part gives a nonlinear correction of the frequency, and the imaginary part produces the nonlinear damping (see Fig. 4).

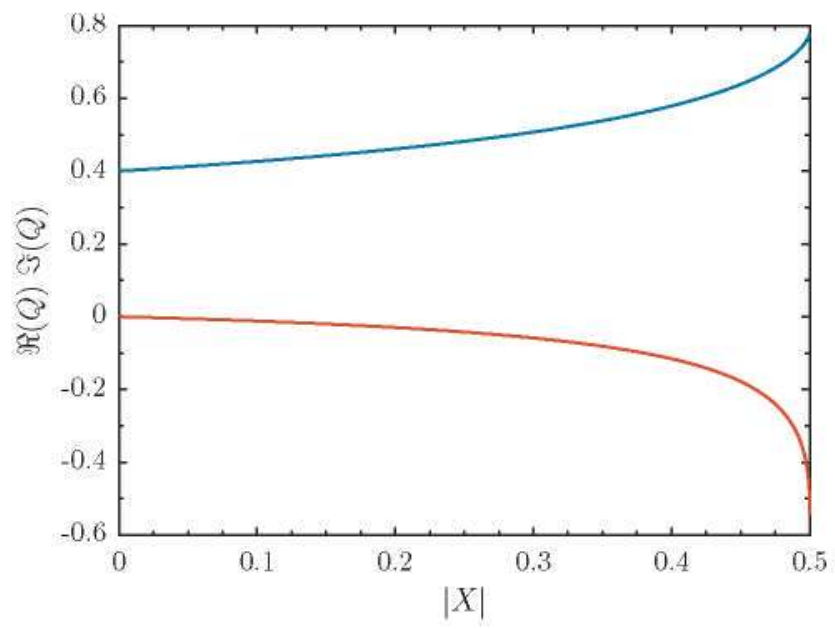

Fig. 4 Complex friction function for the Olofsson microslip model, real part (blue) and imaginary part (red).

The asymptotically reduced order model can be finally formulated as

$$
\begin{aligned}
& 2 \mathrm{i} \frac{d}{d \tau}\left[\begin{array}{c}
X_{1} \\
\vdots \\
X_{j} \\
\vdots \\
X_{N}
\end{array}\right]=\left[\begin{array}{ccccc}
Q\left(\left|X_{1}\right|\right) & & \cdots & & 0 \\
\vdots & \ddots & & & \vdots \\
0 & & Q\left(\left|X_{j}\right|\right) & & 0 \\
\vdots & & & \ddots & \vdots \\
0 & & \cdots & & Q\left(\left|X_{N}\right|\right)
\end{array}\right]\left[\begin{array}{c}
X_{1} \\
\vdots \\
X_{j} \\
\vdots \\
X_{N}
\end{array}\right]+ \\
& {\left[\begin{array}{ccccccc}
-2 k-\mathrm{i} c & k & 0 & & \cdots & & k \\
\vdots & & & & & & \vdots \\
0 & \cdots & k & -2 k-\mathrm{i} c & k & \cdots & 0 \\
\vdots & & & & & & \vdots \\
k & 0 & \cdots & & 0 & k & -2 k-\mathrm{i} c
\end{array}\right]\left[\begin{array}{c}
X_{1} \\
\vdots \\
X_{j} \\
\vdots \\
X_{N}
\end{array}\right]+f\left[\begin{array}{c}
\mathrm{e}^{\mathrm{i} E O \frac{2 \pi}{N}} \\
\vdots \\
\mathrm{e}^{\mathrm{i} E O \frac{2 \pi}{N} j} \\
\vdots \\
\mathrm{e}^{\mathrm{i} E O 2 \pi}
\end{array}\right] \mathrm{e}^{\mathrm{i} \Lambda \omega \tau \tau}}
\end{aligned}
$$

where $X_{j}$ stands for the complex amplitude of the slow modulation of the displacement of the airfoil $j$. The numerical integration of this system requires very low CPU cost because the fast elastic vibration time has been completely filtered out

Several simulations of the full mass-spring model Eq. (4) have been done with the objective of validating the accuracy of the asymptotic model obtained in this section. Numerical calculations have been performed in MatLab, employing a 4th order Runge-Kutta scheme with constant time step. The integration of the mass-spring system is very expensive because the time step has to be small enough to solve the short friction time scale, and the integration time has to be large enough to allow the system to execute many elastic oscillation periods until the stationary state of the blade displacement is reached.

The number of blades is set to $N=13$, the engine order to $E O=3$, the forcing amplitude to $f=0.04$, the stiffness ratio to $k=1$, the mass friction parameter to $\gamma=1$, the material damping $c=0.05$, and the blade to friction stiffness ratio is reduced $\theta=0.1,0.01$, and 0.001 to approach the asymptotic limit $\theta \ll 1$.

The resulting frequency sweeps are presented in Fig. 5, where a clear convergence of the results is obtained as the value of $\theta$ is decreased. In fact, the values for the case $\theta=0.01$ are almost indistinguishable from the ones of the case 
$\theta=0.001$. The asymptotic results from the simplified equation (13) are also depicted, exhibiting an excellent agreement with the cases with $\theta=0.01$ and $\theta=0.001$.

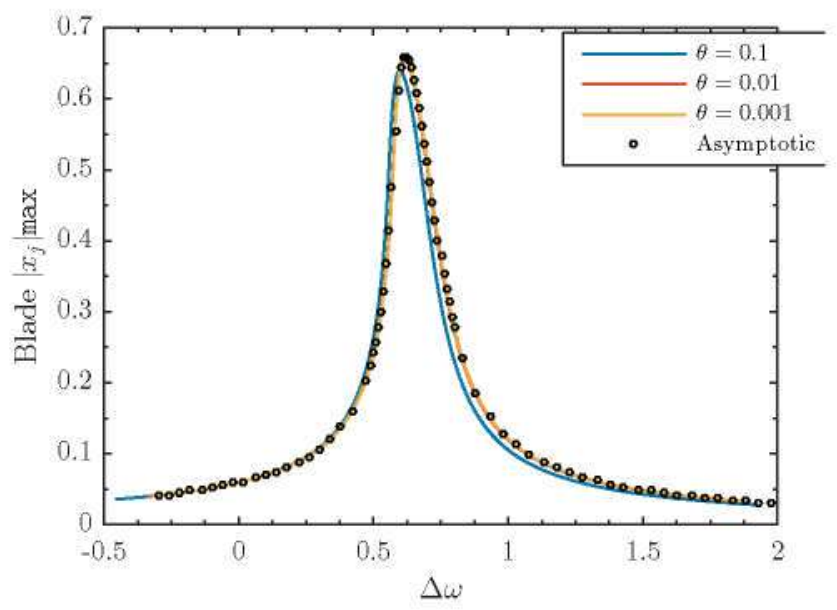

Fig. 5 Forced response of the tuned system.

Figure 6 displays the TW content of the solution at the maximum value reached when sweeping in frequency. As expected in this tuned case, the solution has basically just one single $T W$ with index 3 , which is the TW directly forced by the external forcing (the rest of the TWs exhibit amplitudes below $10^{-4}$ and cannot be appreciated in the plot).
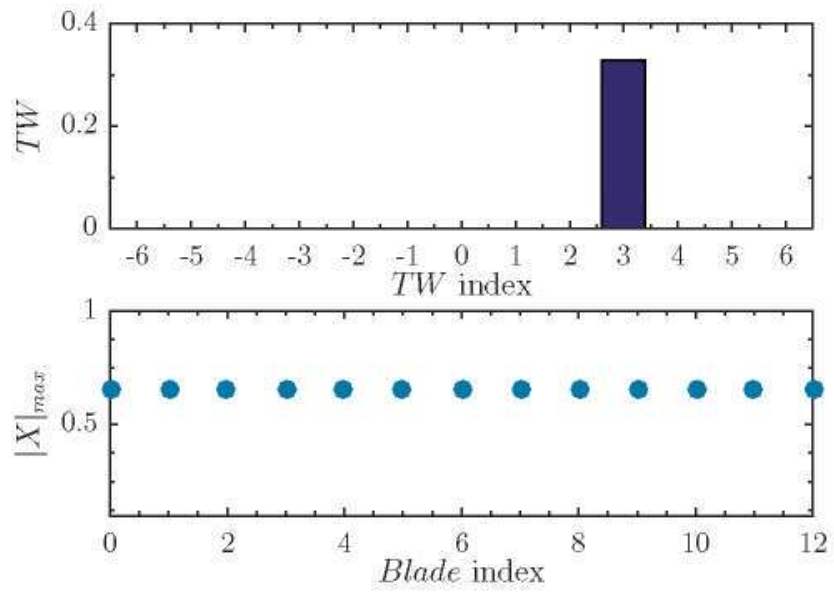

Fig. 6 TW amplitude (top) and blade displacement (bottom) at the point of maximum forced response in Fig. 5.

\section{Mistuned Response}

Mistuning is implemented as a small variation $\theta m_{j}$ of the blade stiffness of each sector. The nondimensional equations for the mass-spring model with mistuning included are given by

$$
\begin{gathered}
\frac{d^{2} \tilde{x}_{j}}{d T^{2}}+\left(1+\theta m_{j}\right)\left(\tilde{x}_{j}-\theta \tilde{y}_{j}\right)+\theta k\left(2 \tilde{x}_{j}-\tilde{x}_{j+1}-\tilde{x}_{j-1}\right)+\theta c \frac{d \tilde{x}_{j}}{d T}=\theta f \mathrm{e}^{\mathrm{i} \tilde{\omega} T+\mathrm{i} E O \frac{2 \pi}{N} j}+\text { c.c. } \\
\theta \gamma \frac{d^{2} \tilde{y}_{j}}{d T^{2}}+\left(\theta \tilde{y}_{j}-\tilde{x}_{j}\right)\left(1+\theta m_{j}\right)+\tilde{F}_{f}\left(\tilde{y}_{j}\right)=0 \quad \text { for } j=1, \ldots, N .
\end{gathered}
$$

The application of the multiple scale method is completely similar to that in the previous section, and the resulting asymptotically reduced order model has just some extra stiffness terms due to mistuning in the diagonal of the last 
matrix:

$$
\begin{aligned}
& 2 \mathrm{i} \frac{d}{d \tau}\left[\begin{array}{c}
X_{1} \\
\vdots \\
X_{j} \\
\vdots \\
X_{N}
\end{array}\right]=\left[\begin{array}{ccccc}
Q\left(\left|X_{1}\right|\right) & & \cdots & & 0 \\
\vdots & \ddots & & & \vdots \\
0 & & Q\left(\left|X_{j}\right|\right) & & 0 \\
\vdots & & & \ddots & \vdots \\
0 & & \cdots & & Q\left(\left|X_{N}\right|\right)
\end{array}\right]\left[\begin{array}{c}
X_{1} \\
\vdots \\
X_{j} \\
\vdots \\
X_{N}
\end{array}\right]+
\end{aligned}
$$

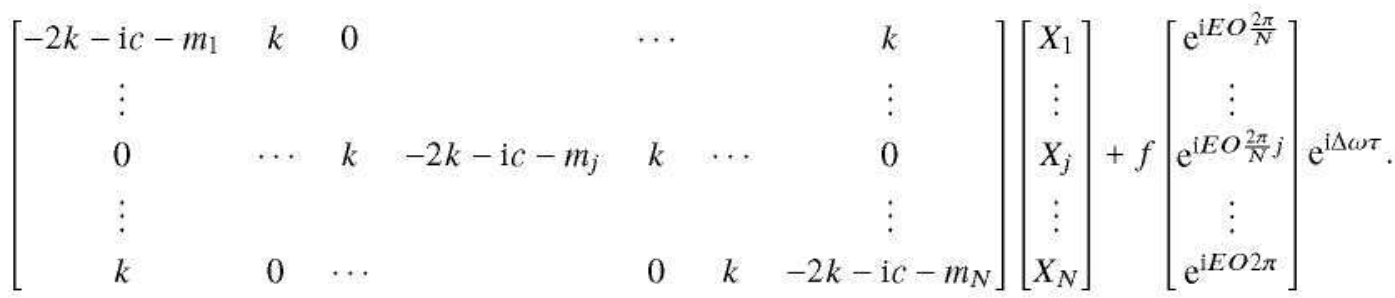

Again, the dynamics of the mass-spring system Eq. (14) is analyzed in the limit of small forcing, small damping (nonlinear friction and material), small ratio of blade and coupling stiffness, and small mistuning effects, and compared with the results form the asymptotic model. Figure 7 displays the response of the mass-spring system for different small values of the parameter $\theta(\theta=0.1, \theta=0.01$ and $\theta=0.001$, ), for a mistuned (randomly distributed) case with the remaining parameters as in the tuned simulations. There is again a clear convergence towards the asymptotic results. As it happened in the tuned case, the plot shows that the cases $\theta=0.01$ and $\theta=0.01$ are almost identical. The usual effect of frequency splitting of the maximum response due to mistuning can be appreciated, together with an amplification (above $25 \%$ ) of the response compared to the tuned case, see Fig. 5.

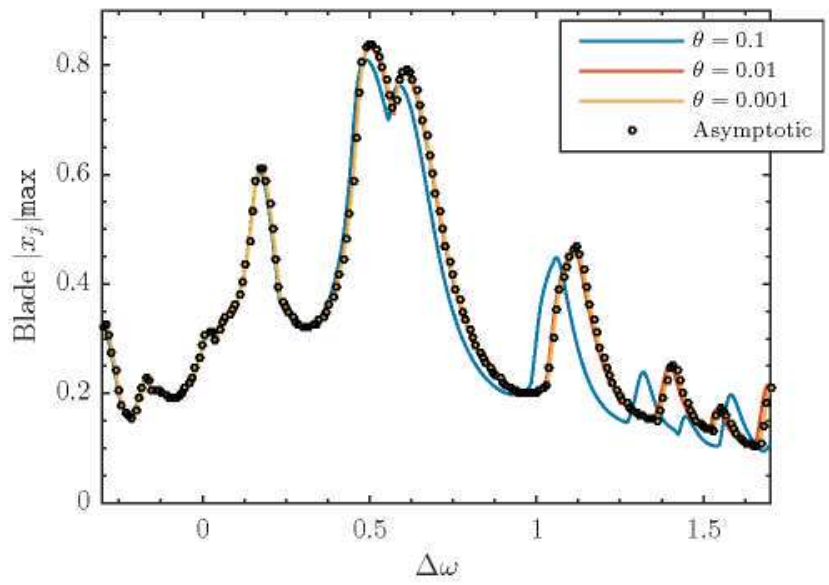

Fig. 7 Forced response of the mistuned system.

Now, we proceed to integrate the system of equations (15) with the aim of examining the combined effect of mistuning and and microslip friction, employing the following parameter values: $N=13, E O=3, f=0.04, k=1$, $\gamma=1, c=0.05$. The mistuning pattern is a the random distribution of the previous simulations, normalized to have maximum amplitude equal to 1 and scaled by a factor settled to be $0 \%$ (tuned case), $40 \%, 80 \%, 120 \%, 200 \%$ and $300 \%$.

Figure 8 depicts the resulting frequency sweeps for the previous values of mistuning intensities. The outcome is similar to the case of constant linear damping [11]: mistuning produces an evident amplification, reaching a $28 \%$ of gain for the case of $100 \%$ mistuning. Although, in the present case, the maximum amplitude does not decay back to the tuned value, instead it remains almost constant for large mistuning values. If mistuning is small ( $40 \%$ f.i.), the maximum response is isolated near the resonance frequency (central large split peaks), but, as mistuning is increased ( $300 \%)$, the response peaks spreads over all the range of frequencies simulated (several peaks with almost same value distributed over the frequency domain).

Now, selecting the maximum response point represented in Fig. 8 for five mistuning cases, $40 \%, 80 \%, 120 \%, 200 \%$ and $300 \%$, we represent the corresponding TW spectrum and blade displacement of the system in Figs. 9, 10, 11, 12, 


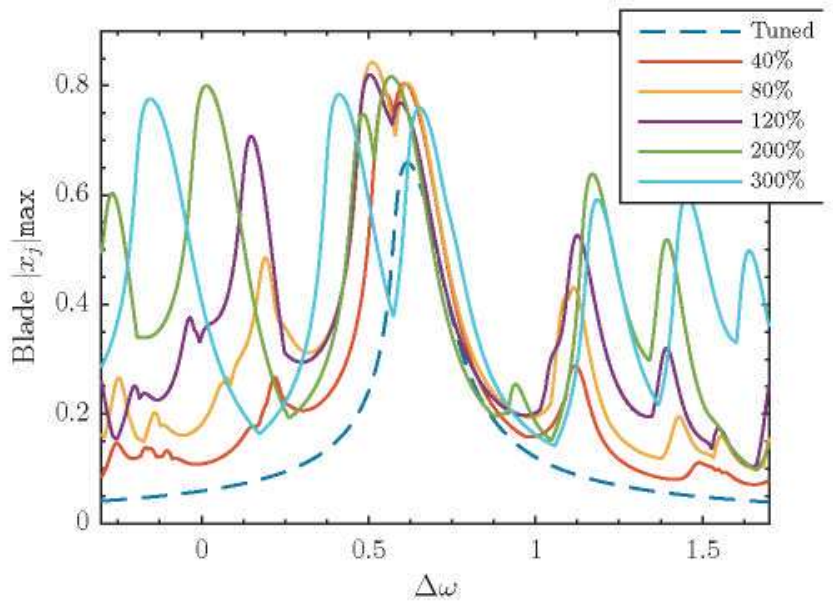

Fig. 8 Forced response of the system for $0 \%$ (tuned case), $40 \%, 80 \%, 120 \%, 200 \%$ and $300 \%$ mistuning.

and 13, respectively. A comparison can be done considering the tuned case illustrated in Fig. 6. The solution now is notably different, as it is no longer composed of just one single $T W$ with index 3 , but another one appears with index -3 , double peak of maximum value response observed in Fig. 7, and remaining almost constant for larger mistuning values. The rest of TWs appear with less intensity (remaining below $10^{-1}$ in Fig. 9) to fill the spectrum as the mistuning is increased, revealing its effect on a family of blade-dominated modes. The results are also significantly different from the case with a ratio between the blade and coupling stiffness $\left(\frac{k_{c}}{k_{b}}\right)$ not small (isolated mode forcing, see [7]), where the response is only composed of the TW with index 3 and -3 , and the all other TW are not excited.
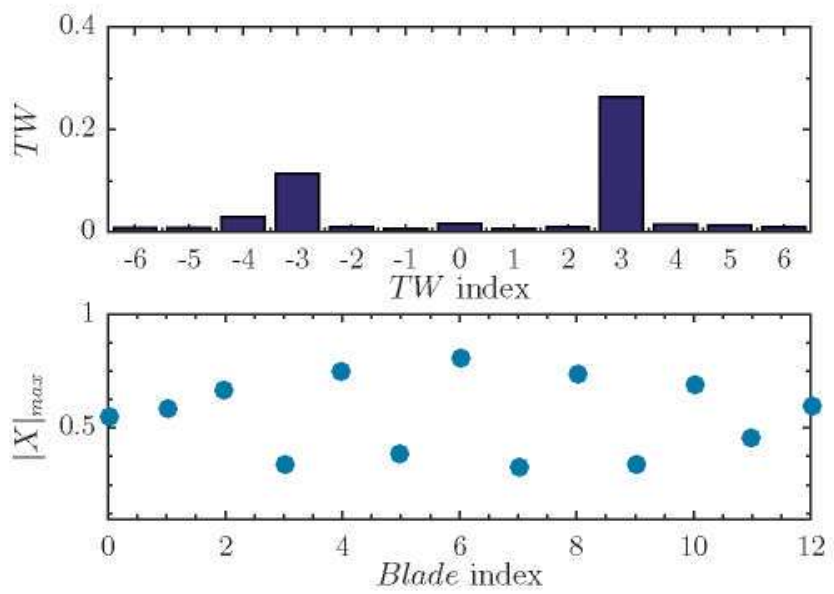

Fig. 9 TW amplitude (top) and blade displacement (bottom) at the point of maximum forced response with $40 \%$ mistuning (see Fig. 8).

Finally, the forced response amplification is presented as function of the scale factor of mistuning in Fig. 14. It can be observed that the maximum amplification appears around $100 \%$ of the mistuned analyzed, corresponding to a gain of approximately $28 \%$, and the maximum amplitude is maintained for larger mistuning values than in the linear damping case.

\section{Conclusions}

In this paper, the asymptotic methodology of multiple scales is applied to the study of the forced response of a mistuned bladed disk in the presence of nonlinear friction (in the microslip regime) for a family of blade-dominated modes.

The ratio of blade stiffness to the microslip friction stiffness $(\theta)$, to the blade coupling stiffness $(\theta k)$, and to the 

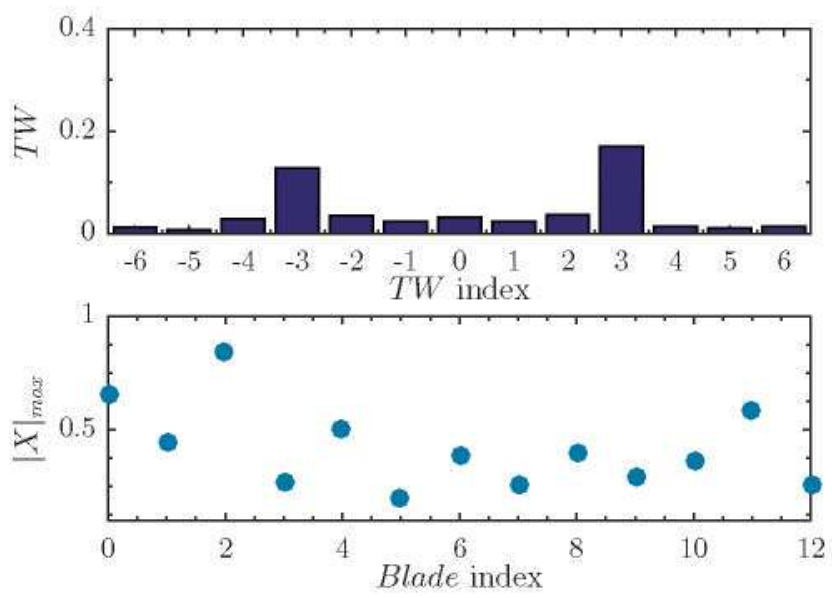

Fig. 10 TW amplitude (top) and blade displacement (bottom) at the point of maximum forced response with $80 \%$ mistuning (see Fig. 8).
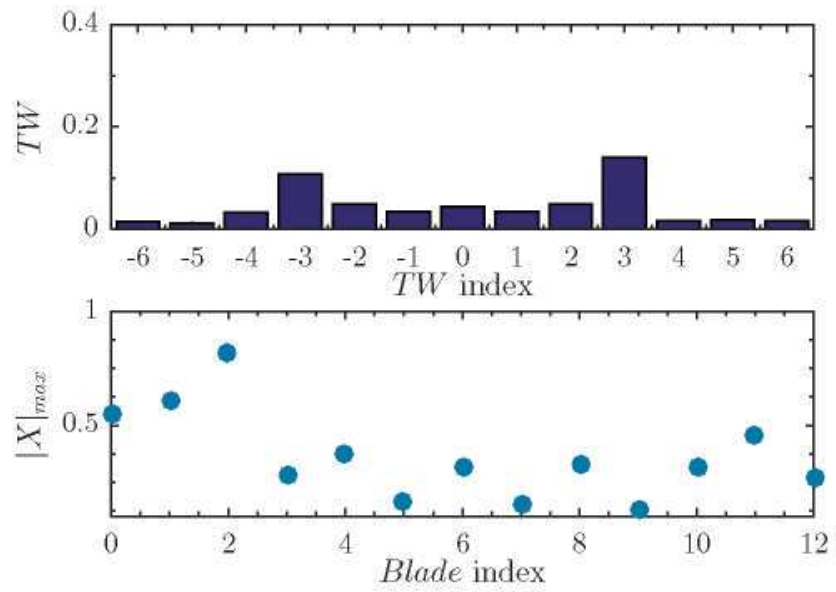

Fig. 11 TW amplitude (top) and blade displacement (bottom) at the point of maximum forced response with $120 \%$ mistuning (see Fig. 8).
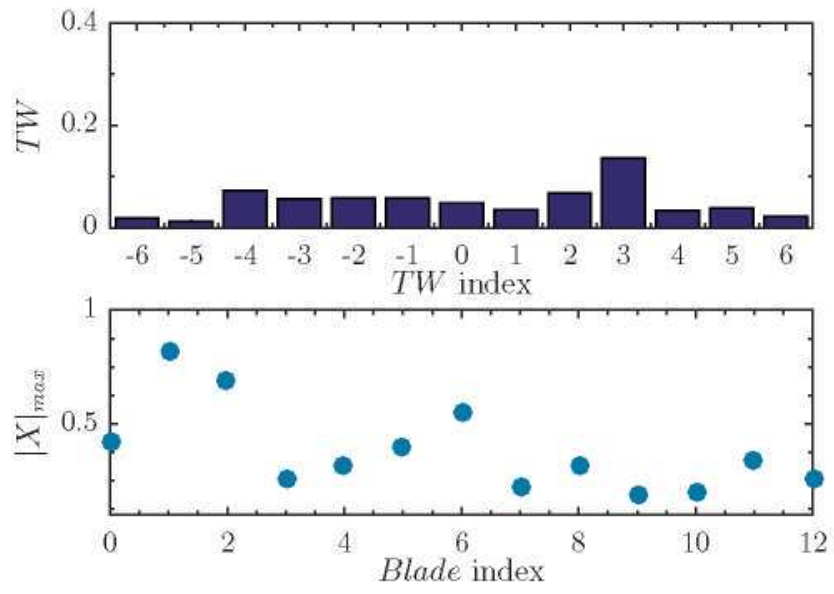

Fig. 12 TW amplitude (top) and blade displacement (bottom) at the point of maximum forced response with $200 \%$ mistuning (see Fig. 8). 

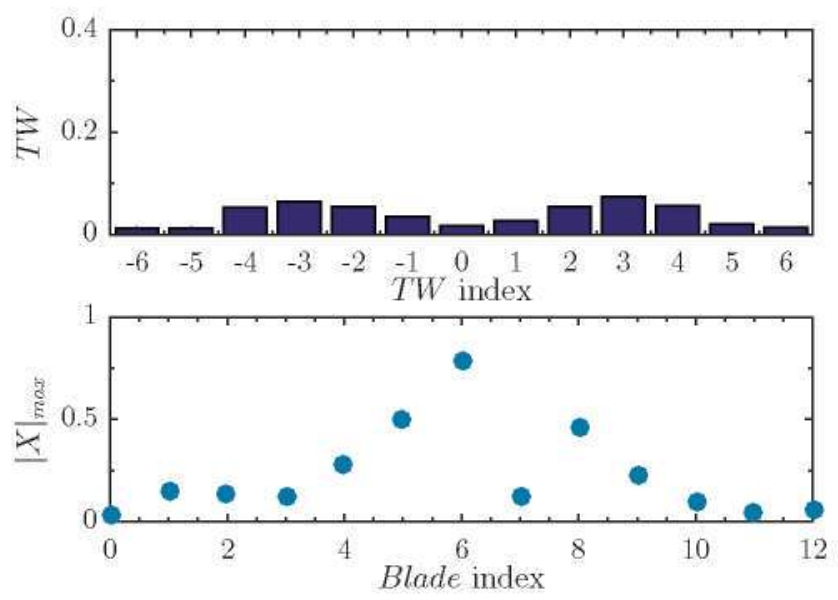

Fig. 13 TW amplitude (top) and blade displacement (bottom) at the point of maximum forced response with $300 \%$ mistuning (see Fig. 8).

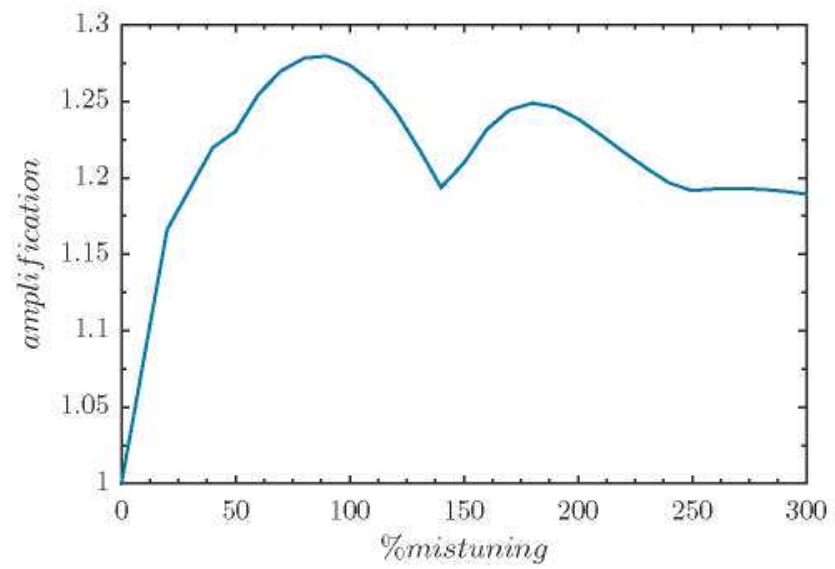

Fig. 14 Forced response amplification variation with mistuning.

stiffenss mistuning $\left(\theta m_{j}\right)$, are all typically small in most realistic configurations. All these small effects develop in a much slower time scale than that of blade elastic vibration, and are captured in the asymptotic reduced model derived in this paper for the limit $\theta \ll 1$.

The integration of the reduced models gives excellent results for values of $\theta=0.01$ and below, and is much less CPU costly than the original equations for the mass-spring models, eqs. (4) and (14), producing a speed-up of the order of 350 times faster.

The study of the mistuned forced response in the presence of nonlinear friction reveals a different behaviour than the one obtained when the linear damping is analyzed. The amplification that the mistuning produces is maintained for large mistuning values, and it does not decay back to the tuned case as it happens in the case of the linear damping ([11]).

\section{Acknowledgments}

This work has been supported by the Spanish Ministerio de Ciencia, Innovación y Universidades under grant DPI2017-84700-R.

\section{References}

[1] Yang, B., and Menq, C., "Characterization of 3D Contact Kinematics and Prediction of Resonant Response of Structures having a 3D Frictional Constraint," Journal of Sound and Vibration, Vol. 217, No. 5, 1998, pp. 909-925. 
[2] Yang, B., Chu, M., and Menq. C., "Stick-Slip-Separation Analysis and Non-Linear Stiffnes and Damping Characterization of Friction Contacts Having Variable Normal Load," Journal of Sound and Vibration, Vol. 210, No. 4. 1998, pp. $461-481$.

[3] Petrov, E. P., and Ewins, D. J., "Analytical Formulation of Friction Interface Elements for Analysis of Nonlinear Multi-Harmonic Vibrations of Bladed Disks," Journal of Turbomachinery, Vol. 125, No. 2, 2003, pp. 364-371.

[4] Petrov. E. P. and Ewins. D. J. "State-of-the-art dynamic analysis for non-linear gas turbine structures," Proceedings of the. Institution of Mechanical Engineers, Part G: Journal of Aerospace Engineering. Vol. 218. No. 3, 2004, pp. 199-211.

[5] Krack, M.. Salles, L., and Thouverez, F., "Vibration Prediction of Bladed Disks Coupled by Friction Joints." Archives of Computational Methods in Engineering, Vol. 24, 2017. pp. 589-636.

[6] Martel, C., Corral, R., and Ivaturi, R., "Flutter Amplitude Saturation by Nonlinear Friction Forces: Reduced Model Verification," Journal of Turbomachinery, Vol. 137, No. 4. 2014. pp. 041004-041004-8.

[7] Martel. C., and Martin. J. A., "Asymptotic description of forced response vibration saturation by friction forces," Proceedings of ASME Turbo Expo 2019: Turbomachinery Technical Conference and Exposition, ASME, GT2019-90756, 2019.

[8] Kevorkian, J.. and Cole. J.. Multiple Scale and Singular Perturbation Methods. Applied Mathematical Sciences. Vol. 114, Springer, 1996.

[9] Bender, C. M., and Orszag, S. A.. Advanced mathematical methods for scientists and engineers: 1 : Asymptotic methods and perturbation theory. Springer, 1999.

[10] Olofsson. U., "Cyclic Microslip Under Unlubricated Conditions," Tribology International, Vol. 28, 1995. pp. $207-217$.

[11] Martel, C. and Corral. R. "Asymptotic Description of maximum Mistuning Amplification of Bladed Disk Forced Response," Journal of Engineering for Gas Turbines and Power, Vol. 131, 2009, p. 022506. 